\title{
Influence of geophysical judgement and software parameters on interpretation of seismic section
}

\author{
VR Balasubramaniam, PC Jha, B Butchi Babu, S Nelliat, YV Sivaram and K Goverdhan \\ National Institute of Rock Mechanics \\ Bengaluru, India \\ vrb.subramanyam@gov.in
}

\begin{abstract}
Seismic refraction has long been adapted for various types site characterization studies. The method has evolved from the classical theory based on Huygens's principle involving straight propagation paths representing spherical wave front heading direction into many novel considerations involving curved-ray-path and cell based finite element techniques for tracing the propagation of seismic waves in the subsurface media and deriving the velocity of the body waves as closely approximated to reality as possible, within the theoretical and computational limits of the routines. In view of multifarious options available in the market for plotting and visualizing and interpretation of seismic sections it becomes important to understand the influence of manual and automated processing techniques on reasonability, accuracy and reliability of velocity sections derived from such techniques. This paper comparatively evaluates the merit and limitation of each of the techniques w.r.t to the site conditions, the depth of investigation and resolution. This study emphasizes the need of optimal selection of field survey and processing parameters with due regard to the constraints experienced during acquisition and processing
\end{abstract}

Keywords-Seismic refraction, conventional techniques, modern techniques, refraction tomography, geophysical judgement, software parameters, impact

\section{INTRODUCTION}

Seismic method is an important geophysical technique due to its near surface imaging capability with high accuracy and reliability [1]. Though seismic methods, whether reflection or refraction, have not been found reliable for exploration of minerals in view of absence of marked definition of the subsurface rocks and disseminated nature of deposits, they are most commonly employed for near surface geological, civil, mining and environmental engineering projects for planning, design, remediation and damage control measures. The economics of implementation of the seismic methods on land make it readily applied for near surface investigations. The seismic methods involve two major categories of investigation viz. surface methods and borehole methods. In this paper surface methods, have been considered for discussion. Two most commonly applied surface methods are 1. Seismic Reflection Method and 2. Seismic Refraction Method. Among the two methods, this paper focuses on the seismic refraction method with the aim of discussing the effect of geophysical judgement, the seismic survey parameters and the processing parameters on the seismic section.

\section{SEISMic Refraction Method}

Seismic Refraction method [1,2,3] have been proven in mapping horizontal interfaces with increasing velocity and depth. Seismic refraction is one of the economical and readily deployable, reliable, geophysical method for data acquisition for near surface investigations in geological, engineering construction and excavation projects. The method is more than 100 years and since then evolved from the fundamental and classical approach based on layered half space approach into ray tracing the refractor based on non-linear travel time theory. [10]. In this section, both the conventional and the recent refraction tomography techniques are discussed.

\section{CONVENTIONAL TECHNIQUES}

The conventional technique is based on seismic waves generated at a point on the surface of the earth propagate down into the earth and get refracted at the interfaces of two consecutive horizontal layers depending upon the angle of incidence (Fig.1). The refraction of seismic waves studied by dividing the subsurface into a number of layers, each having velocities and finite thickness. The strength of the refracted signals depends on acoustic impedance contrast in the medium. The travel times depend on the physical properties of the subsurface rockmass and the attitude of beds [1]. The prime objective of the refraction technique is to determine information about the nature of the subsurface soil or rock, their attitude based on variation of seismic signal amplitude and frequency.

The velocity and thickness of the subsurface are modified in forward modeling to obtain the closest traveltime and thus, get the best fit of time slope that would resemble the layered model as per the basic theoretical assumption. The normal plot of the arrival times of the refracted seismic waves is as shown in (Fig. 2). The critical time corresponding to the critical distance and the intercept time are the most common and fundamental parameters determined from the field data.

Conventional techniques for processing seismic refraction first arrivals use delay time, plus-minus or generalized reciprocal method [1]. These Techniques make 


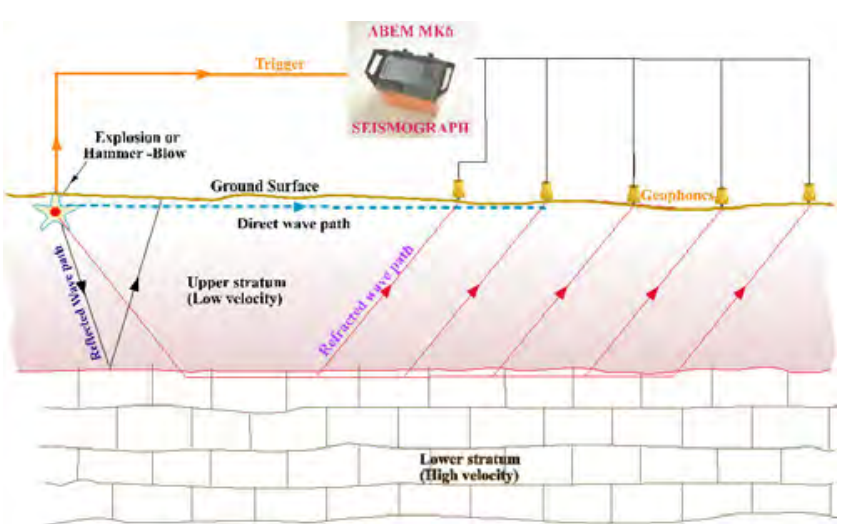

Fig.1 Schematic layout of seismic sensors array and refraction from subsurface horizons

simplifying assumptions on the subsurface layer horizons based on heterogeneity, lateral or depth-wise changes in rock properties. The conventional methods are founded on the fundamental principles of wave propagation obeying Huygen's principle and Snell's law. The general output of the travel time plot is shown in the Fig. 2.

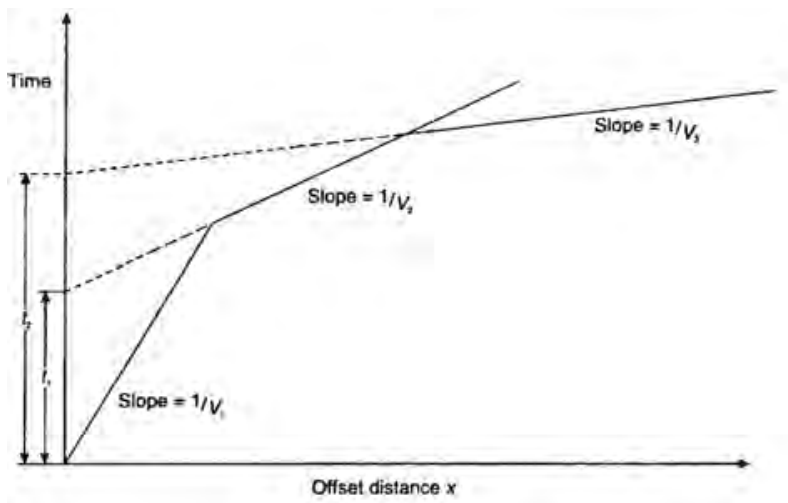

Fig.2 Typical graphical output of first arrival times from horizontal interfaces

Generally, interpretation of travel time is based on this basic curve type looking for identification of different subsurface horizons. [5]. Such conventional plotting to derive subsurface horizon depth works out reasonably well when the layers are horizontal and the velocity increases with depth. Mapping of travel-time curves to refractors is a difficult task when considering the actual geological situations. For example, extremely undulating terrain conditions in mountains might imply abrupt changes in the subsurface, such as very thin layer of soil / weathered rock, dipping planes / joints or voids that vary drastically wrt the assumption of horizontal layers with increasing velocity.

There are some slightly advanced conventional techniques like Generalized Reciprocal Method (GRM), Plus-Minus Method [6] and Travel-Time Continuation Method. These methods have partly graphical and partly empirical approach to determining the dip of a horizon or sudden changes in time of arrival at a station point. Despite, such endeavor these techniques are never closer to defining the attitudes reliably. Thus, it may be safely be concluded that they are reliable in gentle topographic and subsurface variations.

\section{MODERN APPROACH - REFRACTION TOMOGRAPHY}

With advances in instrumentation, data acquisition technique and data processing, the two methods are capable of high-resolution imaging based on curved ray propagation and inversion techniques, and have been increasingly applied to near-surface engineering and environmental investigations. This capability has led to high-resolution imaging of the subsurface, very thin layers, homogeneous rockmass, dipping interfaces, weak or fractured rock, minor faults, shear zones, vertical contacts, undulating interfaces, subsidence, buried channels, near surface voids, or cavities. The progress of computer technology has simplified calculus procedures, thereby leading to a great development in geophysical data processing methodologies, particularly for application to soil studies regarding civil engineering infrastructure. The amazing increase in microcomputer processing velocity during the last few years, added to their huge storage capability, has enabled the development of algorithms from different theoretical backgrounds, giving birth to refraction tomography [8].

Unlike conventional refraction methods, seismic refraction tomography (SRT) does not require that the model be broken into continuous layers having constant velocity. Instead, the model is made up of many small constant velocity grid cells or nodes. Inversion is performed by an automated procedure involving raytracing through an initial mode and comparing the modelled travel-times to the field data, and adjusting the model grid-by grid to match the modeled travel-times to these. This process is iteratively repeated until a preset number of iterations has been reached. Because there is no assumption of continuous constant velocity layers, SRT can model localized velocity anomalies. Refraction tomography has capability to overcome the limitations encountered with the conventional techniques like very thin layer sandwiched between two high velocity layers, gradual change in velocity, lateral changes, compact and loose zones within a medium.

For example, the tomographic inversion used in the Rayfract [6] is based on physically realistic modeling of first break propagation for P-wave and S-wave surveys. The refraction times are forward modeled [5] and the travel time residuals are projected back along the wave paths instead of conventional rays. This increases the numerical robustness of the inversion. A 1D model is determined from the seismic travel-time data by horizontally averaging $\Delta \mathrm{t}-\mathrm{V} 1 \mathrm{D}$ velocitydepth profiles [6] along the seismic line. The starting model is then refined with 2D WET Wave path Eikonal Travel time inversion [4]. The curved-ray path inversion method adapted $[9,10]$, in the software thus delivers a continuous depth vs velocity profiles along the seismic line. With this kind of handling of the arrival time data, geological settings could be brought out in the seismic section in a more realistic manner, including pitchouts and outcrops.

Travel times can be traced to obtain refractor points either interactively or semi-automatically based on apparent 
Common-Mid-Point (CMP) velocities and refractor velocity intervals. Interactive or seismic automatic picking is always reliable as the fully automatic picking sometimes shifts the first arrival time, more frequently on a noisy trace. The travel time is finally processed based on CMP intercepttime-refraction technique, Plus-Minus and Wave front refraction technique on 2D WET tomograms to map picked travel-times to refractors. There is also an inbuilt option for checking the picked travel times for reciprocal travel-time errors and for shifting picks for all traces of same shot with trigger delay correction. The processing technique should be chosen according to the terrain conditions in the field and based on expected or mapped subsurface geological trend. This will enable optimal application of processing parameters for the set of travel-times gathered, leading to more realistic mapping to the refractor depth.

Secondly, the type of coverage of seismic profiles plays another crucial role in mapping of the refractors. Generally, a good coverage involves forward and reverse shooting at more than half the spread lengths as well as at closer distances, to have the wave paths arriving from deeper as well as shallower refractors. However, sometimes the refraction profile spread is restricted to low coverage lengths due to site conditions. For such low coverage spreads, overlapping of the spread by half-length would help avoid "edge-effects" in processing, rendering more close to reality depth mapping to refractors. In order to arrive at reliable depth determination, it is highly useful to have at least one borehole on the line of seismic survey in order to calibrate the depths of refractors.

\section{DISCUSSION OF OUTPUT CONVENTIONAL AND RECENT TECHNIQUES}

The results of the of processing by both the conventional and the tomography methods are presented in this section. The Fig. $3(\mathrm{a}, \mathrm{b} \& \mathrm{c})$ and the Fig.4 (a, b \& c) show the conventional plot ( $3 \mathrm{a}$ and $4 \mathrm{a}$ ) of arrival times for processing in two cases by conventional techniques[13]. The figures at $3 \mathrm{~b}$ and $4 \mathrm{~b}$ show the depth of two different velocity horizons $(600 \mathrm{~m} / \mathrm{s} \& 1100 \mathrm{~m} / \mathrm{s}$ and $600 \& 1800 \mathrm{~m} / \mathrm{s})$ calculated from conventional processing techniques. The figures at $3 \mathrm{c}$ and $4 \mathrm{c}$ show the one obtained through CMP travel time processing. The final output section was otbtained based on WET Eikonal tomography [11], inversion to trace the travel time to the refractor. From the two types of sections it can be clearly seen that the section obtained from the conventional processing shows depth of horizons obtained from calculation at discrete points. All the discrete points are then connected for continuity of that horizon. In case of refraction tomography, small cells are generated by dividing the initial model for a given spread length and the expected depth of propagation. The travel time propagation through these cells generate individual velocity cells, and connecting these cells lead to generation of continuous velocity mapping all through the section.

This contrast in the output is quite indicative of the kind of influence they would have on the geophysical judgement and interpretation of the subsurface layers. The conventional ones lead to interpretation of two horizons as obtained from the slope of the time-distance curve, while the continuous velocity mapped in the tomographic section renders an option of fixing the proper horizon based on additional information in the form of geological and geotechnical observations. Thus, the seismic section from the tomographic inversion has the edge of closest possible mapping of the depth of different rock types based on the velocity gradient observed in the section.

While there are a set of assumptions in conventional techniques posing limitation on the processing of travel times and the computation of accurate seismic velocities that lead to a geophysicist's bias and judgement, there is also a share of disadvantages in using refraction tomography software, particularly in selecting automated or semiautomated parameter selection for processing the travel times. The automatic picking of travel times itself is not an obvious solution to quickly arriving at the realistic travel times, as would have been experienced by most of the practicing geophysicist.

Thus, manual intervention for determining the correct arrival time is a necessity. This involves personal judgement and experience of a geophysicist to precisely estimate the travel time in noisy data or haphazard arrivals experienced in highly undulating steep slopy terrains
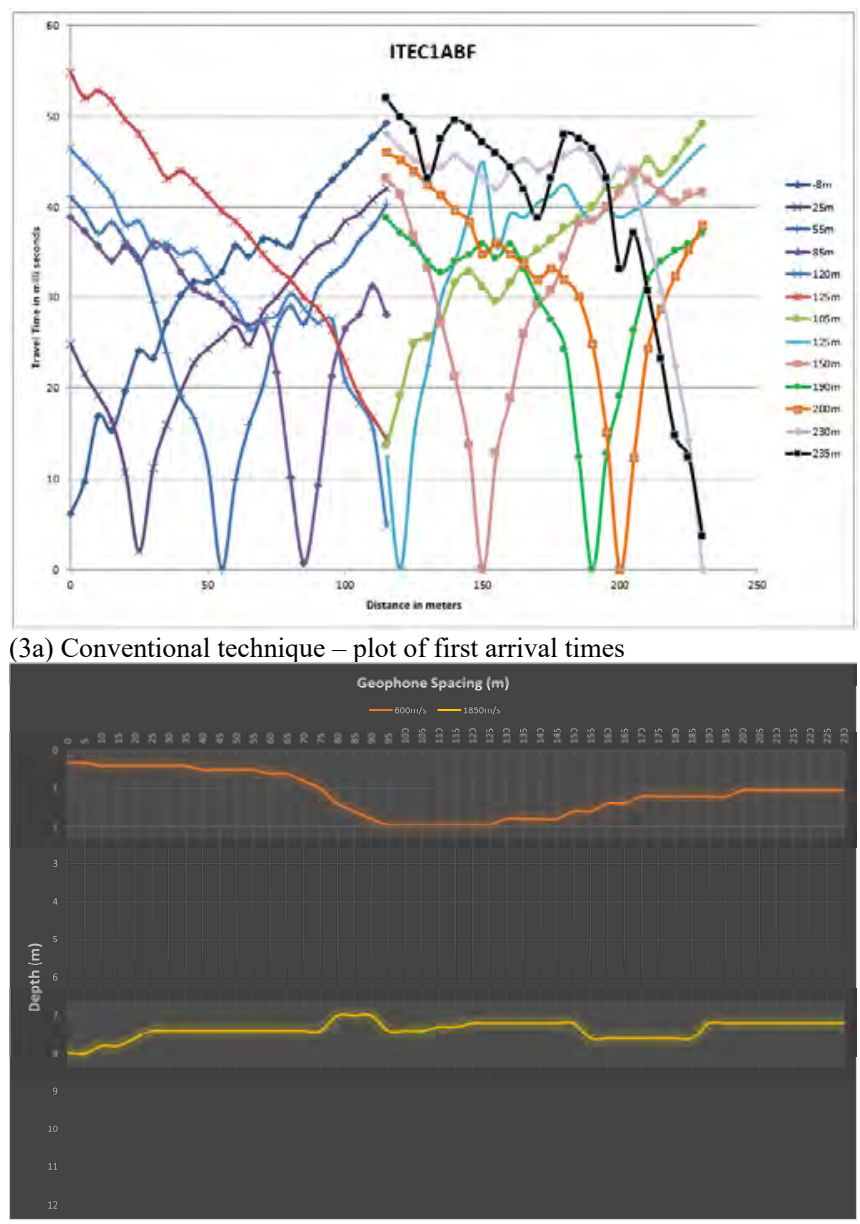

(3b) Seismic section from conventional technique 


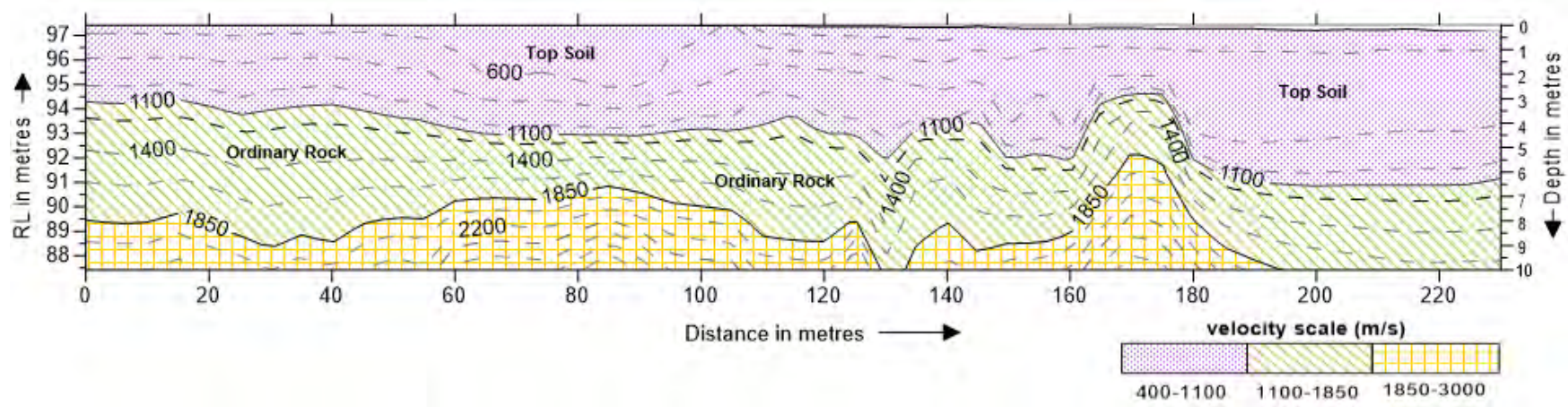

(3c) Typical seismic section from WET tomography

Fig. 3 Outcome of conventional and recent processing techniques

Judging the reliable time needs a thorough understanding of the spherical wave propagation through the subsurface rock mass, nearby interfering structures, the reflection, refraction and diffraction phenomena that the spherical wave has undergone through the propagation path and other ambient noises at the site.

Some of the significant parameters that require diligent attention of the geophysicist while feeding the input for processing are:

1. The accuracy of survey parameters that includes coordinates of sensors and source locations, geophone spacing, shot distance, number of shots per seismic data set, noise pre-filter setting and definition of arrival times selection. Checking the consistency of the survey parameters for a given seismic array, including offset distances of sensors and source locations, is essential for setting next stage of processing parameters.

2. The refraction tomography software build up an initial model [4] based on inputs of survey geometry and preliminary top layer velocity (weathering velocity) and subsequent layers. The velocity estimates are to be determined by verifying the arrival times manually at different points of the seismic array. This plays a significant role in mapping the top layer velocity and the depth of refractors.

3. The length of the seismic spread, coverage type and number of shot points influence in properly training the

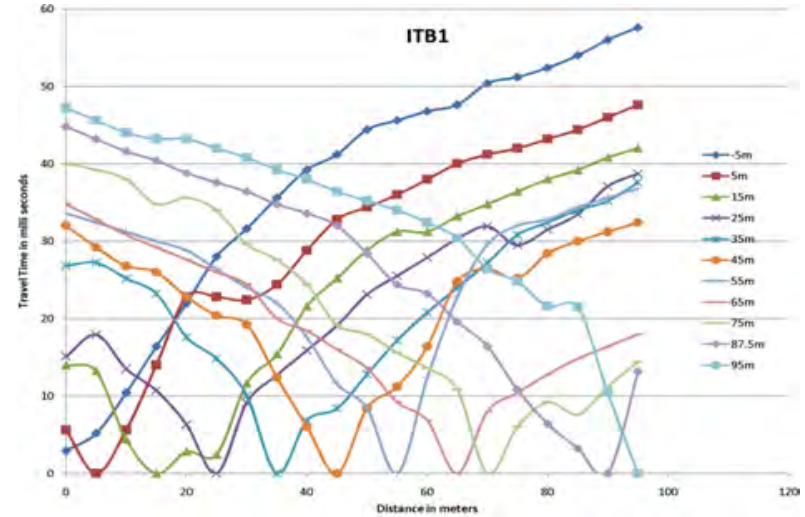

(4a) Conventional technique - plot of first arrival times



(4b) Seismic section from conventional technique



(4c) Typical seismic section from WET tomography

Fig.4 Outcome of conventional and recent processing techniques 
initial model during the processing stage for arriving at reasonable estimates for inverted velocity values.

4. Correction or refinement of the initial model based on time to depth conversions and resulting refractor velocities; fixing of the minimum and maximum propagation velocities from the observed field truths and that determined from simple calculations based on geological inputs, as tomography software routines rely on accurate estimates of near surface velocity.

5. Type of inversion technique chosen for a given type of terrain and the seismic spread chosen to gather seismic data like regression over number of receivers, linear or non-linear regression [7], how many receiver stations are covered in each set of neighboring common midpoints, static corrections that are consistent with the terrain topography and datum plane.

\section{CONCLUSION}

Geophysical judgement involves from the planning stage of a seismic refraction survey based on the geological conditions and the terrain type. The selection of seismic array and data acquisition procedure affects the seismic data quality. In addition, field truths observed need to be well correlated with the geological data and the acquired seismic data. Any decision taken should be thoroughly subject to geophysical scrutiny vis-a-vis the objective as they influence the geophysical processing and interpretation. Proper selection of survey parameters by detailed understanding of the scope and limitations of the theory adapted in terms of various processing routines is a fundamental requirement to effectively use the refraction tomography technique for interpretation of seismic travel times and section generated based on them. Limiting factors of tomography inversion routines like number of shots, number of traces per record, source-receiver geometry, semi-automatic or manual intervention options for improving section accuracy, sampling accuracy are to be remembered while employing software routines.

Systematic following of the survey and processing stage factors and whole some understanding of the acquisition to interpretation stage geophysical parameters that affect the seismic data would help optimize the use of recent tools for reliable seismic imaging of the subsurface.

\section{ACKNOWLEDGMENT}

The authors sincerely thank Director, National Institute of Rock Mechanics for according permission to publish the work and this paper in the conference. Colleagues at NIRM who were an inspirational support in completing the manuscript in shortest possible time are also thankfully remembered.

\section{REFERENCES}

[1] J. R. Pelton, in D.K.Butler. Near surface geophysics, Investigations in Geophysics, 13, SEG, 732., 2005.

[2] W.M Telford, LP,Geldart, R.E Sheriff. Applied Geophysics, Cambridge university press $2^{\text {nd }}$ ed., 792,1990
[3] B. Redpath, Seismic refraction for engineering site investigation: Explosives Excavation Research Lab., TR E- 73-4, 51p. 1973.

[4] Palmer, D., The generalized reciprocal method of seismic refraction interpretation: Society of Exploration Geophysics, 104p. 1980.

[5] Dobrin, B. M. and Savit, C. H. Introduction to Geophysical Prospecting. Intl. Edition, McGraw-Hill, 867. 1988

[6] Hagedoorn, J.G., The plus-minus method of interpreting seismic refraction sections: Geophysical Prospecting, 7, 158-1821959.

[7] G.T.Schuster and A Quintus-Bosz. Wavepath eikonal traveltime inversion : Theory. Geophysics, volume 58, p. 1314-1323., 1993.

[8] Sheehan J.R., Doll W.E. and Mandell W. 2005. An Evaluation of Methods and Available Software for Seismic Refraction Tomography. Journal of Environmental and Engineering Geophysics, volume 10, p. 21-34. JEEG March 2005 issue. ISSN 1083-1363.

[9] Siegfried R. Rohdewald, The Deltat V 1D method for seismic refraction inversion: Theory, Publn of Intelligent Resources Inc

[10] B.S. Gibson, M.E. Odegard and GH. Sutton. Nonlinear least-squares, inversion of traveltime data for a linear velocity-depth relationship. Geophysics, volume 44, pp.185-194., 1979.

[11] I. Lecomte " Improving modelling and inversion in refraction seismics with a first-order" Eikonal solver. Geophysical Prospecting, volume 48, p. 437-454. 2000

[12] NIRM Technical Reports (unpublished), 2006-2013.

[13] Siegfried Rohdewald "Interpretation of First-Arrival Travel Times with Wavepath Eikonal Traveltime Inversion and Wavefront Refraction" 24rd EEGS Symposium on the Application of Geophysics to Engineering and Environmental Problems, 10 April 2011 